\title{
Planificación y uso del tiempo académico asincrónico de estudiantes universitarios en condiciones de pandemia
}

\author{
Carolina Zambrano ${ }^{1 *}$, Ingrid Bravo ${ }^{2,4}$, Jorge Maluenda-Albornoz ${ }^{3}$, y Valeria A. Infante-Villagrán ${ }^{4}$ \\ (1) Facultad de Educación, Departamento de Metodología de Investigación e Informática Educacional, \\ Universidad de Concepción, Concepción, Chile. (correo-e: carolinazambrano@gmail.com) \\ (2) Facultad de Educación y Ciencias Sociales, Pedagogía en Educación Diferencial, Universidad Adventista de Chile, \\ Chillán, Chile. (correo-e: ingridbravo@unach.cl; ingridspring@gmail.com) \\ (3) Facultad de Ingeniería, Unidad de Educación en Ingeniería, Universidad de Concepción. Barrio Universitario s/n, \\ Concepción, Chile. (correo-e: jorgemaluendaa@gmail.com) \\ (4) Facultad de Ciencias Sociales, Departamento de Psicología, Doctorado en Psicología, Universidad de Concepción. \\ Barrio Universitario s/n, Concepción, Chile. (correo-e: valeria.a.infante.v@gmail.com) \\ * Autor a quien debe ser enviada la correspondencia
}

Recibido Feb. 4, 2021; Aceptado Abr. 5, 2021; Versión final Abr. 30, 2021, Publicado Ago. 2021

\begin{abstract}
Resumen
El objetivo de este estudio fue analizar la planificación y uso del tiempo académico asincrónico de estudiantes universitarios de carreras del área de educación, psicología e ingeniería. La muestra ( $n=73$ ) fueron estudiantes que pertenecen al $15 \%$ de los mejores promedios de nota de su grupo en condiciones de pandemia COVID-19. Se utilizó un registro de planificación semanal del estudio que permite obtener información respecto de las variables de: propósito del estudio, actividad de estudio, asignaturas que estudia y uso del tiempo semanal asincrónico. Los resultados mostraron que las actividades de estudio que predominan son: lectura, elaboración de información y organización de información. Se advierte poco tiempo para la preparación del estudio y poca autorregulación en la etapa de disposición al estudio. El promedio de horas de estudio asincrónicas fue de 2,9 horas al día. En conclusión, se sugiere orientar el trabajo de docencia online hacía la inclusión de estrategias de evaluación formativa, planificación y gestión del tiempo.
\end{abstract}

Palabras clave: autorregulación; aprendizaje; disposición; estudio; propósitos; estrategias; COVID-19

\section{Planning and use of asynchronous academic time by university students during the pandemic}

\begin{abstract}
The main objective of this study was to analyze the planning and use of asynchronous academic time by university students enrolled in education, psychology, and engineering degrees. The sample $(n=73)$ was comprised by student with the best average grades in their groups during the COVID-19 pandemic. That is, students who belonged to the top $15 \%$ of their class. A weekly study planning record was used to collect data regarding the following variables: study purpose, study activity, study subject, and use of asynchronous weekly time. The results showed that the most predominant study activities were reading, information preparation, and information organization. There was also scarce preparation time for studying and for self-regulation during the getting-ready for studying stage. The average asynchronous study hours were 2.9 hours per day. In conclusion, it is suggested to focus online teaching towards the inclusion of formative evaluation strategies, planning, and time management.
\end{abstract}




\section{INTRODUCCIÓN}

La suspensión de clases presenciales en Chile y en parte del mundo producto de la pandemia por Covid 19 ha evidenciado la necesidad de un rol activo por parte de los estudiantes. En este contexto, la gestión del tiempo y la planificación del estudio son temas clave y comportamientos estratégicos que podrían permitir al estudiante mejores resultados académicos en un ambiente de educación online en que existe mayor flexibilidad horaria y se espera autocontrol del estudiante en su proceso educativo (Baars et al., 2015; Pérez et al., Cobo et al., 2018). Asimismo, se espera la autorregulación de su propio aprendizaje (Delen et al., 2014; Liaw y Huang, 2013). Por lo anterior, en la presente investigación se busca estudiar de forma preliminar un aspecto de la planificación y uso del tiempo académico de estudiantes universitarios que presentan buen rendimiento académico en condiciones de pandemia. El criterio para establecer el buen rendimiento se basa en el promedio obtenido durante el primer semestre del año 2020 cuando se presenta la condición de educación a distancia producto de la pandemia.

Para estudiar la planificación y uso del tiempo académico asincrónico de estudiantes universitarios en condiciones de educación online por pandemia Covid-19 se usa como marco teórico el aprendizaje autorregulado (Zimmerman y Labuhn, 2012). El aprendizaje autorregulado es un proceso multidimensional desarrollado por los estudiantes cuando estos tienen conciencia de sus propios procesos cognitivos, socioafectivos y motivacionales (Boekaerts y Corno, 2005; Pintrich, 2000). Para la autorregulación del aprendizaje se han propuesto varios modelos con respaldo teórico y empírico (Hadwin y Oshige, 2011; Zimmerman y Labuhn, 2012; entre otros). Los autores coinciden en que la autorregulación del aprendizaje es un proceso cíclico y se consideran tres fases: disposición, desempeño y evaluación (Panadero, 2017). La primera fase, de disposición o preparación, implica un proceso de activación, análisis de tareas, planificación, establecimiento de objetivos y metas. La segunda fase de desempeño considera la ejecución de la tarea. Finalmente, en la fase de evaluación el estudiante regula y adapta su actuación (Panadero, 2017; Puustinen y Pulkkinen, 2001; Zimmerman y Labuhn, 2012).

En particular, la primera fase de disposición al estudio consiste en que el estudiante formule objetivos, analice las tareas y genere una planificación para realizarlas. Esto es, que organice sus tiempos para las actividades que requiere efectuar para poder desempeñarse de la mejor forma. En esta fase de disposición al estudio se conjugan una serie de factores tales como: habilidad del estudiante para analizar sus tareas, nivel de motivación, autoeficacia e interés en cada tarea (Zimmerman y Labuhn, 2012). La autorregulación del aprendizaje favorece la motivación al aprendizaje, es así como los estudiantes que poseen altos niveles de estrategias de disposición al estudio presentan creencias positivas de su propia capacidad para regular su proceso de disposición al estudio (Zimmerman y Labuhn, 2012).

Asimismo, atribuyen las causas de sus éxitos al esfuerzo, disminuyen las explicaciones de fracaso académico a falta de capacidad o causas externas. La disposición al estudio es un elemento importante para el éxito académico, incluye comportamientos de estructuración del tiempo, planificación de tareas, cumplimiento de la planificación establecida, gestión de las relaciones sociales y actividades de ocio. En este contexto, los estudiantes con alto rendimiento consideran que pueden gestionar su tiempo de forma adecuada, obteniendo beneficios en términos de resultados de su rendimiento y evitando la presión derivada de contar con un cúmulo de tareas pendientes no abarcables. Los estudiantes con bajo rendimiento poseen un nivel menor de autoconfianza en cuanto a la gestión del tiempo, asumen su mala gestión del tiempo y la imposibilidad de organizar adecuadamente sus actividades (Panadero, 2017; Puustinen y Pulkkinen, 2001; Zimmerman y Labuhn, 2012).

Por los antecedentes anteriores, esta investigación preliminar tiene como objetivo estudiar cómo planifican su tiempo los estudiantes universitarios que pertenecen al grupo mejor evaluado cuando se presentan condiciones de educación a distancia. Cabe destacar que se analizó la planificación del estudio que realizan los estudiantes de forma autónoma y asincrónica sin considerar al trabajo sincrónico en las plataformas online. Así, se buscó describir la planificación y el uso del tiempo semanal de estudio usando variables tales como: propósito del estudio, actividad de estudio, asignaturas que estudia y uso del tiempo semanal asincrónico. Esta información puede aportar al profesorado y los estudiantes para que realicen procesos de planificación del estudio en sus asignaturas con el propósito de mejorar la capacidad de autorregulación y autonomía centrada en el aprendizaje que es imprescindible en la educación a distancia.

En suma, la investigación busca responder a las siguientes preguntas: 1) ¿cómo planifican su tiempo académico a sincrónico una muestra de estudiantes universitarios que pertenecen al grupo mejor evaluado cuando se presentan condiciones de educación a distancia?; 2) ¿qué actividades de estudio realizan?; y 3) ¿qué propósitos de estudio tienen?. A continuación, se muestra el proceso de disposición al estudio con el subproceso de planificación. 


\section{Disposición al estudio}

La fase de disposición al estudio es la primera etapa del ciclo de autorregulación del aprendizaje, se divide en 5 subprocesos que se muestran en la Figura 1. Según Zimmerman y Labuhn (2012) los procesos de disposición al estudio son: i) Activación: subproceso en el cual el estudiante se prepara y dispone a estudiar, implica iniciativa personal y perseverancia y altos niveles de automotivación. ii) Análisis de tareas: subproceso asociado a la actividad en la que el estudiante realiza un análisis de la tarea que debe desarrollar, implica la división de la tarea de aprendizaje en componentes clave y pasos específicos para resolverla. iii) Planificación: subproceso en que el estudiante realiza una planificación sobre cómo llevará a cabo la tarea. Aquellas y aquellos estudiantes que son proactivos tienen a tener una planificación más eficaz, eligen estrategias para mejorar su cognición, controlan su afecto y dirigen su ejecución motora. iv) Establecimiento de objetivos: subproceso en que el estudiante se plantea sus objetivos, definiéndolos de manera concreta y acorde a la tarea que intenta resolver. v) Metas: subproceso en el cual el estudiante define sus metas y/o propósitos. Cuando las y los estudiantes presentan habilidades superiores de análisis de la tarea, los alumnos se fijan metas claras y alcanzables.

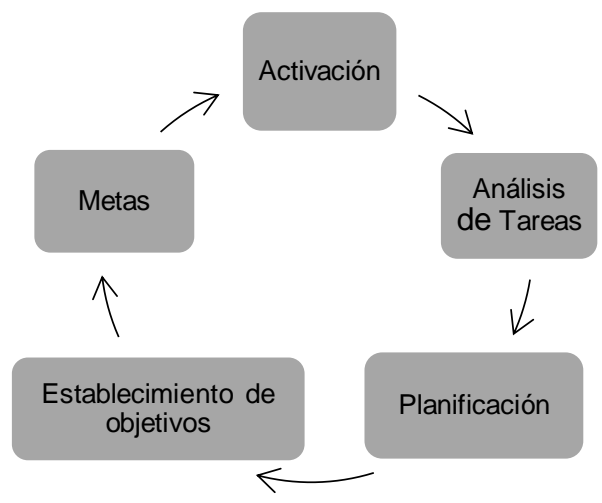

Fig. 1: Proceso de disposición al estudio (Traducido de Zimmerman y Labuhn, 2012)

Para estudiar la planificación del estudio de forma empírica desde una parte del marco teórico del proceso de disposición al estudio (Zimmerman y Labuhn, 2012) se requiere adquirir datos de estudiantes respecto de su planificación semanal. Para ello, se analizan registros de planificación semanal del estudio de estudiantes que cumplen con la condición de buen rendimiento académico. El registro de planificación semanal del estudio incluye las siguientes variables: propósito u objetivo académico del estudio, actividad de estudio específica realizada, asignatura(s) a la(s) que aporta la actividad y tiempo total usado para el estudio (minutos).

\section{METODOLOGÍA}

En este apartado se presenta detalle sobre el diseño de la investigación, sobre el instrumento utilizado, sobre las variables, los participantes, y finalmente sobre los procedimientos de análisis.

\section{Diseño}

Se empleará un diseño exploratorio y descriptivo usando una matriz de registro de actividades académicas de una semana (ver Tabla 1) que es llenada por los estudiantes que cumplen con el requisito de pertenecer al $15 \%$ mejor evaluado de su grupo.

\section{Instrumento}

El instrumento utilizado fue una matriz de registro de actividades académicas de una semana (ver Tabla 1) que permite registrar el tiempo total de estudio, el propósito u objetivo de estudio, la actividad de estudio realizada y la asignatura a la que aporta la actividad de estudio. En la Tabla 1 se observa la matriz de registro de actividades académicas para una semana (día 1 al día 7).

Tabla 1: Matriz de registro de actividades académicas para una semana de estudio

\begin{tabular}{|c|c|c|c|c|}
\hline Día & $\begin{array}{c}\text { Tiempo total usado para } \\
\text { el estudio (minutos) }\end{array}$ & $\begin{array}{c}\text { Propósito u objetivo } \\
\text { de estudio }\end{array}$ & $\begin{array}{c}\text { Actividad de estudio } \\
\text { específica realizada }\end{array}$ & $\begin{array}{c}\text { Asignatura(s) a la(s) que } \\
\text { aporta la actividad. }\end{array}$ \\
\hline Día 1 & & & & \\
\hline$\ldots$ & & & & \\
\hline ... & & & & \\
\hline Día 7 & & & & \\
\hline
\end{tabular}




\section{Variables}

Las variables son: i) Tiempo total usado para el estudio: se mide en minutos totales de estudio que declara el estudiante al ingresar la información en la matriz de registro de actividades académicas para una semana de estudio. ii) Propósito u objetivo académico: se refiere a los propósitos de estudio que el estudiante declara. Por ejemplo: preparar y ordenar el material de mis asignaturas para clases siguientes, preparar una evaluación, entre otros. iii) Actividad de estudio: describe la actividad de estudio que el estudiante realiza. Por ejemplo: realizar un mapa conceptual, leer la materia, preparar una presentación de una tarea, entre otros. iv) Asignatura(s) a la(s) que aporta la actividad de estudio: especifica la o las asignaturas que el estudiante indica que estudia.

\section{Participantes}

Participan 73 estudiantes de 5 universidades: Universidad Adventista, Universidad Católica de Temuco, Universidad de Concepción, Universidad San Sebastián y Universidad Santa María. Un 64.38\% pertenecen a carrera de Educación, $24.65 \%$ pertenecen a carrera de ciencias sociales y un $10.95 \%$ a carrera de ingeniería. La técnica de muestreo es no probabilística por conveniencia ya que los estudiantes que participaron fueron escogidos de forma intencionada según su rendimiento académico porque pertenecen al $15 \%$ mejor evaluado de su grupo durante el primer semestre del año 2020 cuando se presentan condiciones de pandemia covid-19.

\section{Procedimiento de análisis}

La matriz para el registro de actividades académicas de una semana fue registrada en Excel por cada uno de los estudiantes y luego se analizó sintetizando los resultados a través de estadística descriptiva y análisis de contenido (Andréu, 2001) para la categorización de la variable propósito de estudio y la categorización de la variable actividad de estudio.

\section{RESULTADOS}

Los resultados se ordenan en cinco subsecciones. Primero la caracterización de la muestra, segundo los resultados respecto del tiempo de estudio asincrónico semanal. Un tercer apartado con los propósitos de estudio categorizados, un cuarto apartado con las actividades de estudio categorizadas. Y finalmente, la cantidad de asignaturas estudiadas por día.

\section{Caracterización de la muestra}

La Tabla 2 presenta la muestra agrupada por Área, dependencia del establecimiento educacional de enseñanza secundaria (Municipal, Particular Subvencionado, Particular), edad y sexo. Se observa solo 3 participantes que pertenecen a establecimiento particular pagado lo que hace referencia a la alta segregación del sistema educativo chileno (Canales, Bellei y Orellana, 2016; Valenzuela, Bellei y de los Ríos, 2014). Sistema educativo en el cual sólo entre el $5 \%$ y $6 \%$ de los estudiantes pueden asistir a un establecimiento particular pagado (Bellei, Orellana y Canales, 2020). La baja cantidad de estudiantes en el área Ingeniería se debe a la condición de pertenecer al 15\% mejor evaluado de su grupo. Y en el caso de la alta frecuencia de mujeres en el ámbito de carreras del área de educación y ciencias sociales se debe a que son carreras que han tenido una evolución hacia un alto porcentaje de ingreso de mujeres llegando en ocasiones a cubrir más del $90 \%$ de la matricula por parte del género femenino.

Tabla 2: Caracterización de la muestra

\begin{tabular}{|l|c|c|c|c|c|c|c|c|}
\hline Área & Frecuencia & \multicolumn{2}{|c|}{ Edad } & \multicolumn{3}{c|}{ Dependencia } & \multicolumn{2}{c|}{ Sexo } \\
\hline & & $\mathrm{M}$ & $\mathrm{DS}$ & Municipal & $\begin{array}{c}\text { Particular } \\
\text { subvencionado }\end{array}$ & $\begin{array}{c}\text { Particular } \\
\text { pagado }\end{array}$ & $\begin{array}{c}\text { Frecuencia } \\
\text { Hombres }\end{array}$ & $\begin{array}{c}\text { Frecuencia } \\
\text { Mujeres }\end{array}$ \\
\hline $\begin{array}{l}\text { Área Ciencias } \\
\text { Sociales }\end{array}$ & 18 & 20.39 & 2.06 & 5 & 10 & 3 & 4 & 14 \\
\hline Área Educación & 47 & 21.73 & 2.79 & 5 & $\mathbf{4 2}$ & 0 & 0 & 47 \\
\hline Área Ingeniería & 8 & 22 & 1.51 & 4 & 4 & 0 & 4 & 4 \\
\hline Total & 73 & & & 14 & $\mathbf{5 6}$ & 3 & 8 & $\mathbf{6 5}$ \\
\hline
\end{tabular}

\section{Tiempo de Estudio}

En la Figura 2 se observa el tiempo de estudio promedio a la semana medido en minutos que fue indicado por los estudiantes, es el tiempo de estudio asincrónico, es decir, lo que los estudiantes dedican de forma particular y autónoma a sus actividades académicas. El gráfico muestra la tendencia en el tiempo de estudio 
durante una semana. En general el tiempo de estudio promedio es similar durante la semana, observando que disminuye durante el sábado y domingo, lo que podría advertir hábitos de estudio favorables en estudiantes que presentan buen rendimiento académico durante el primer semestre del año 2020. Luego, al realizar la conversión a horas de estudio asincrónicas a la semana se observa que el promedio es de $\mathrm{M}=2.58$ horas al día de lunes a domingo y $\mathrm{M}=2,9$ horas al día si se considera el promedio de lunes a viernes.

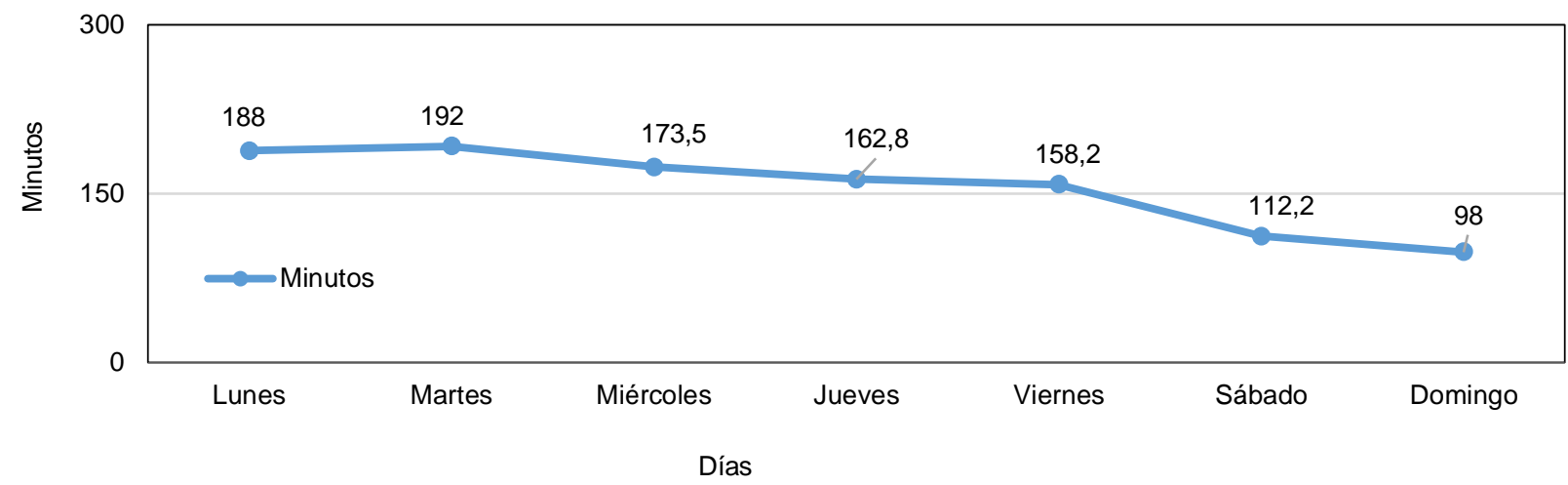

Fig. 2: Gráfico de tendencia de minutos de estudio por día de la semana

Luego, la Figura 3 representa la cantidad de estudiantes por rangos de hora promedio de estudio a la semana. Se observa que 19 estudiantes señalan estudiar en el rango de 15 a 19,9 horas de estudio a la semana. Para los rangos mayores a 25 horas de estudio a la semana se observa que pocos estudiantes registraron esos tiempos de estudio.

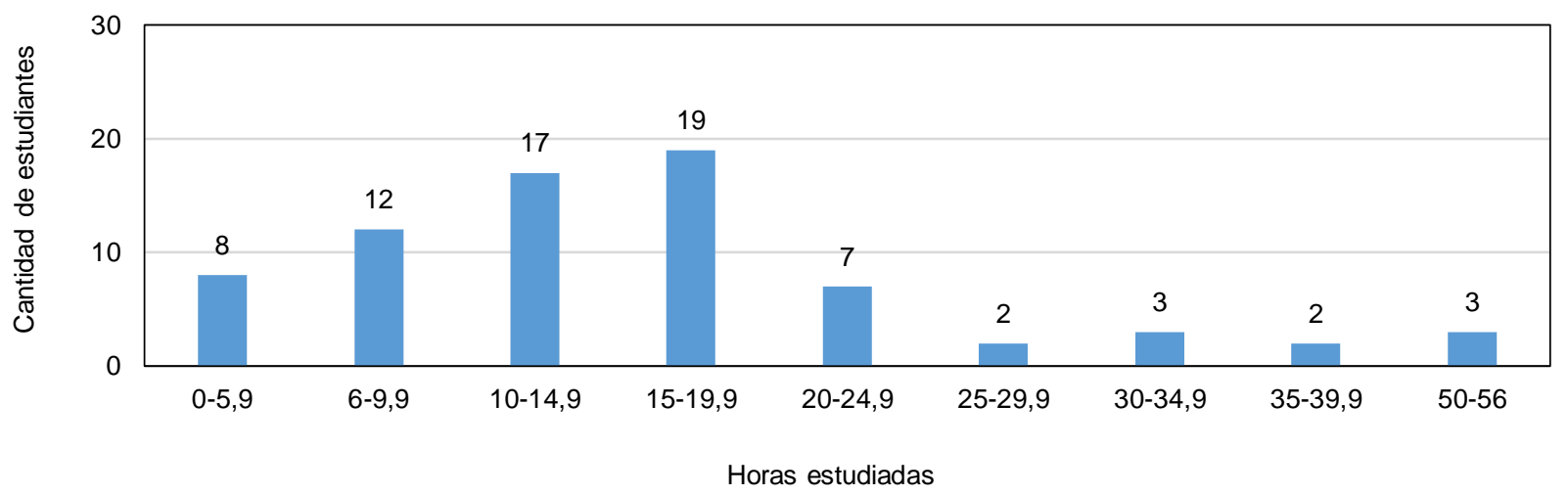

Fig. 3: Cantidad de estudiantes por horas promedio de estudio a la semana

Finalmente, en la Figura 4 se muestra el tiempo promedio de estudio a la semana (medido en horas a la semana) según año en la carrera. Se observa que los estudiantes de primer año son los que más tiempo señalaron estudiar con $M=21,8$ horas a la semana versus estudiantes de último año donde disminuye a la mitad el tiempo de estudio $M=10,2$ horas a la semana.

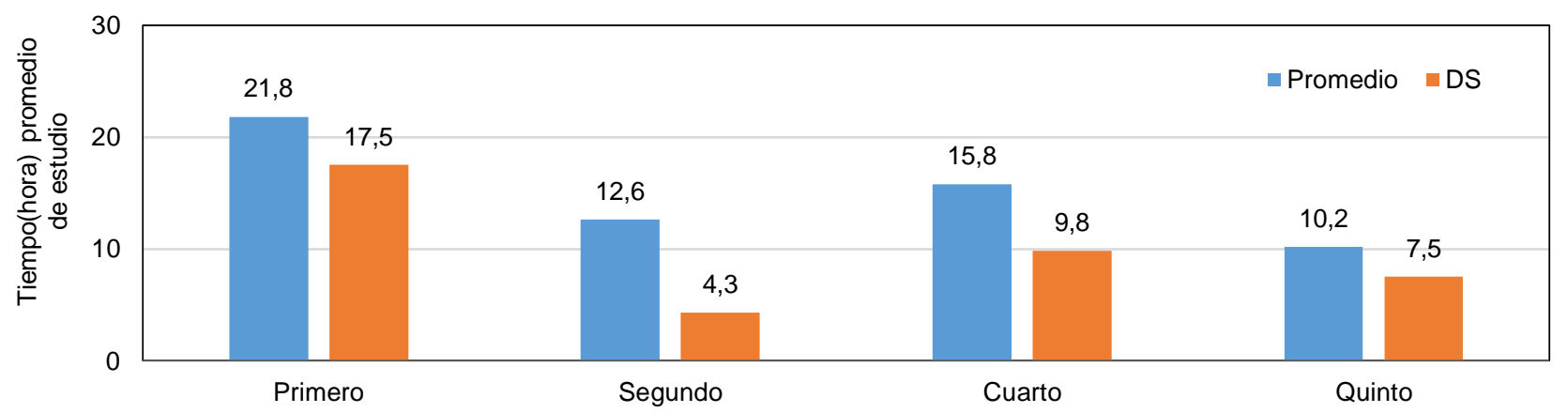

Año en la carrera

Fig. 4: Tiempo promedio de estudio a la semana según año en la carrera 


\section{Propósitos de Estudio}

En esta sección se presentan los resultados asociados a la variable propósito de estudio. Esta variable es cualitativa y se estudió desde los registros de planificación de los estudiantes mediante un análisis de contenido. Como resultado del análisis de contenido se establecieron tres categorías que emergen desde los datos entregados por los estudiantes para los propósitos de estudio, se explican a continuación: 1) Actividad de estudio: se refiere a que el estudiante señala como propósito de estudio actividades de ejecución del estudio sin indicar que la actividad que realiza tiene como propósito preparar una evaluación. Por ejemplo, resumir la materia. 2) Preparación al estudio: se relaciona con actividades que tienen como propósito preparar el ambiente y/o el material antes de realizar la actividad de estudio. Por ejemplo, ordenar los apuntes de las clases. 3) Actividad de estudio para una evaluación: son actividades que tienen como propósito preparar una evaluación. Por ejemplo, repasar la materia para el certamen escribiendo un resumen. En la Tabla 4 se muestran los porcentajes por categoría de propósito de estudio según el día de la semana. Se puede observar que el mayor porcentaje de tiempo se utiliza con el propósito de realizar una actividad de estudio.

En la Figura 5 se observa el gráfico con los porcentajes de uso del tiempo según los propósitos declarados por los estudiantes para cada día de la semana. Es claro que el propósito de ejecutar una actividad de estudio predomina todos los días. También, es interesante observar que los días sábado y domingo el porcentaje de tiempo en que no se estudia es elevado y se advierte que podría ser un patrón de habito de estudio favorable debido al perfil del estudiante que participo en el estudio que corresponde al $15 \%$ mejor evaluado de su grupo y que podría tener un mayor autocontrol de su actividad académica por un ambiente favorable al estudio. Estas sugerencias deben ser profundizadas en nuevos estudios.

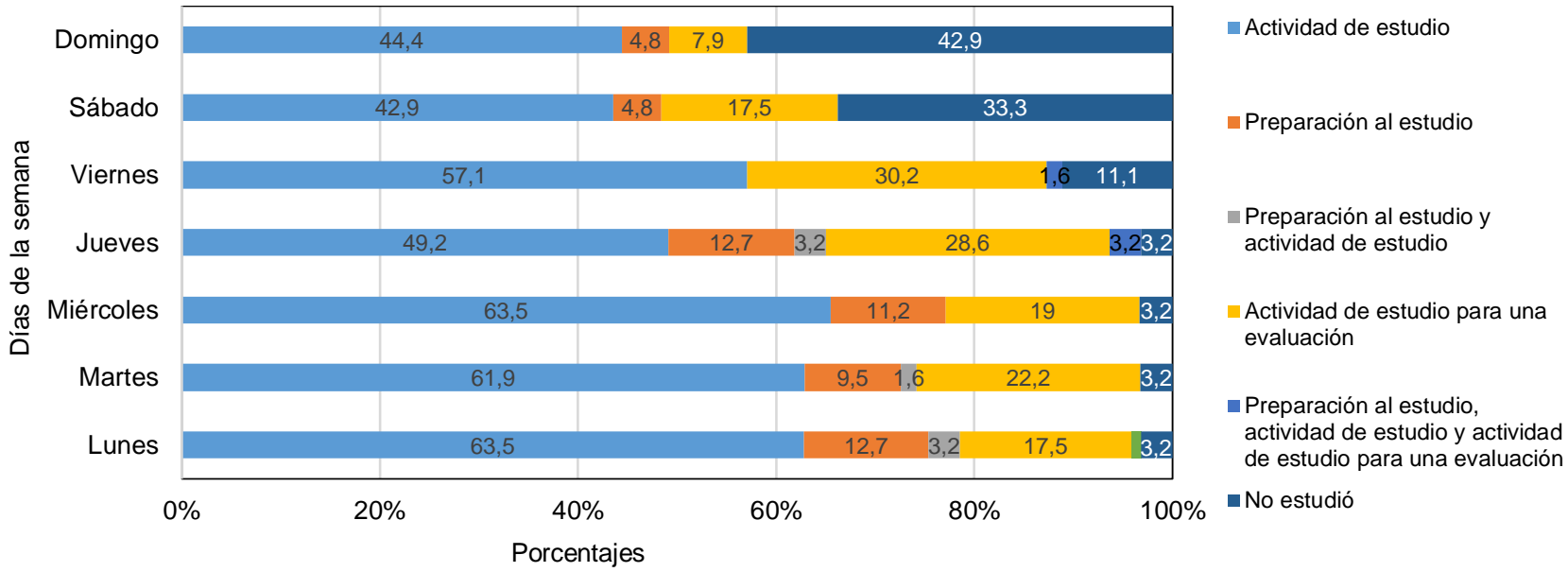

Fig. 5: Porcentajes por categoría de propósito según el día de la semana

Finalmente, en la Figura 6 se muestra un gráfico con el porcentaje promedio semanal por categoría de propósito de estudio. Se observa que el propósito de estudio que mayor porcentaje presenta es la actividad de estudio que se refiere a la ejecución del estudio. Para la preparación al estudio, se advierte un bajo porcentaje de tiempo.

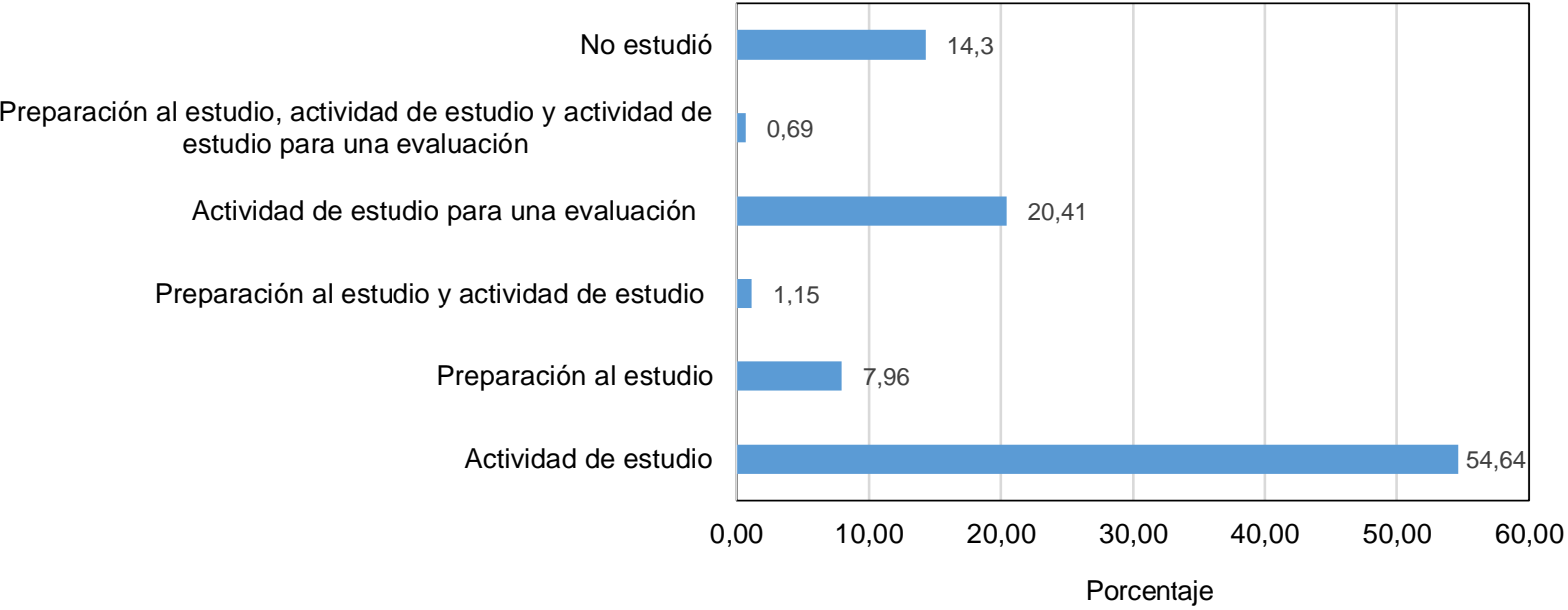

Fig. 6: Porcentaje promedio semanal por categoría de propósito de estudio 


\section{Actividades de estudio}

En este apartado se presentan los resultados asociados a la variable actividades de estudio. Esta variable es cualitativa y se estudió desde los registros de planificación de los estudiantes mediante un análisis de contenido. Como resultado del análisis de contenido se establecieron seis categorías que emergen desde los datos. Las categorías para las actividades de estudio se explican a continuación: 1) Lectura= son actividades de estudio que involucran la lectura de los contenidos por parte de los estudiantes. Por ejemplo, leer la materia para repasar. 2) Revisión de videos= son actividades donde el estudiante señala que revisa videos para estudiar. Por ejemplo, revisar los videos para repasar los contenidos. 3) Elaborar información= se refiere a actividades de estudio en que el estudiante indica aplicar acciones para elaborar información para estudiar. Por ejemplo, crear un resumen. 4) Organizar información= se refiere a actividades de estudio en que el estudiante indica aplicar acciones de organización de información para estudiar. Por ejemplo, crear un mapa conceptual. 5) Responder preguntas de evaluación= son actividades de estudio en que los estudiantes responden a preguntas que han sido dejadas como tareas por sus profesores. Por ejemplo: Responder a las preguntas disponibles en el material de las presentaciones. 6) Planificar= son actividades en que el estudiante indica que realiza un proceso de planificación del estudio, 7) Búsqueda de información= son actividades de estudio en que el estudiante indica que realiza búsquedas de información adicional al material o contenidos entregados por el profesor, 8) Trabajo grupal en línea= son actividades de estudio online en grupo organizadas por los mismos estudiantes, 9) Sin actividad= se refiere al caso en que el estudiante no registro actividad de estudio.

En la Figura 7 se muestra los porcentajes por categoría de actividades de estudio según el día de la semana. Se observa que la actividad de estudio que predomina es la lectura, seguida por la elaboración de información. Situación que da cuenta del proceso de lectura y posterior resumen de los contenidos cuando se estudia. Luego, la Figura 8 muestra los porcentajes promedio por categoría de actividad de estudio durante una semana.

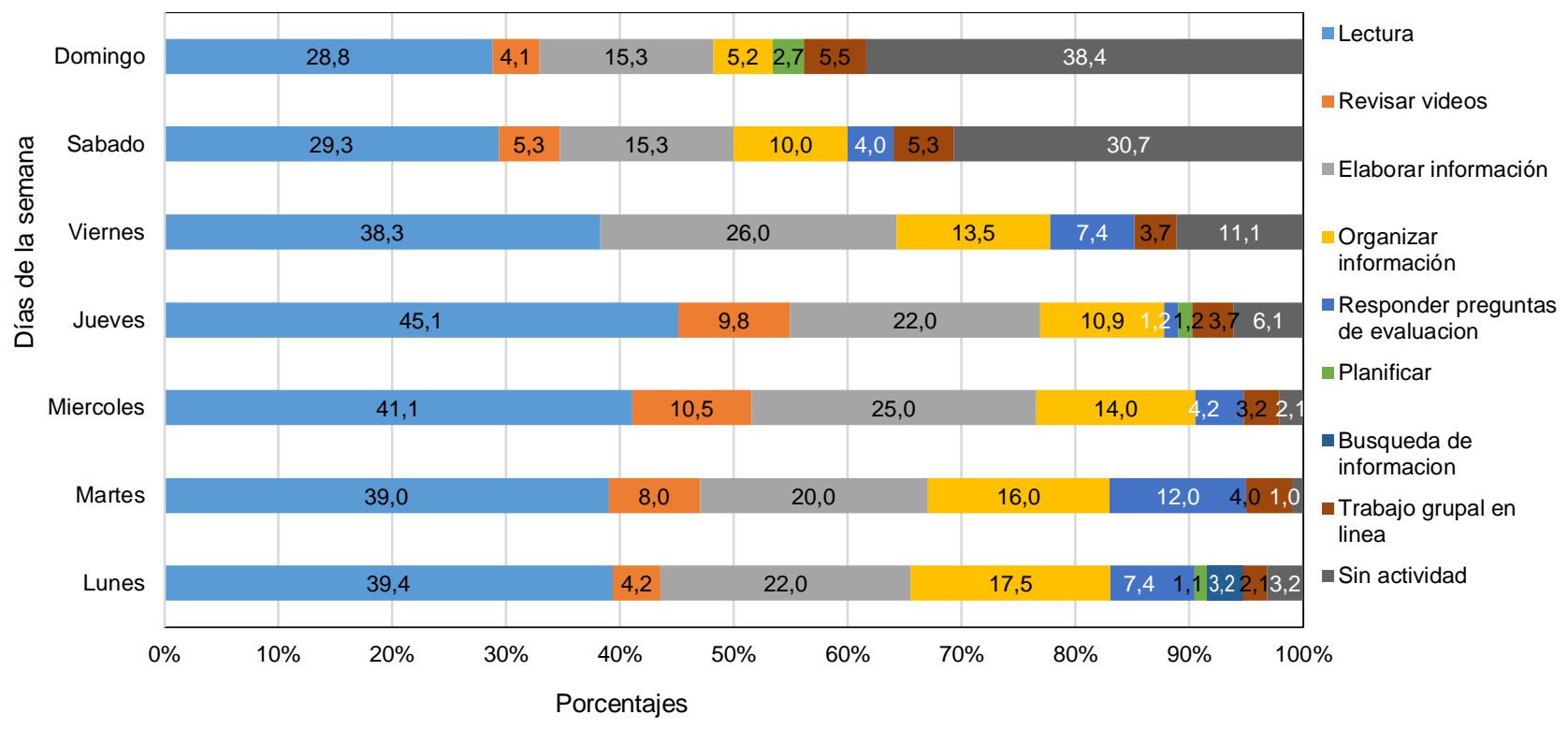

Fig. 7: Porcentajes por categoría de actividad de estudio según día de la semana

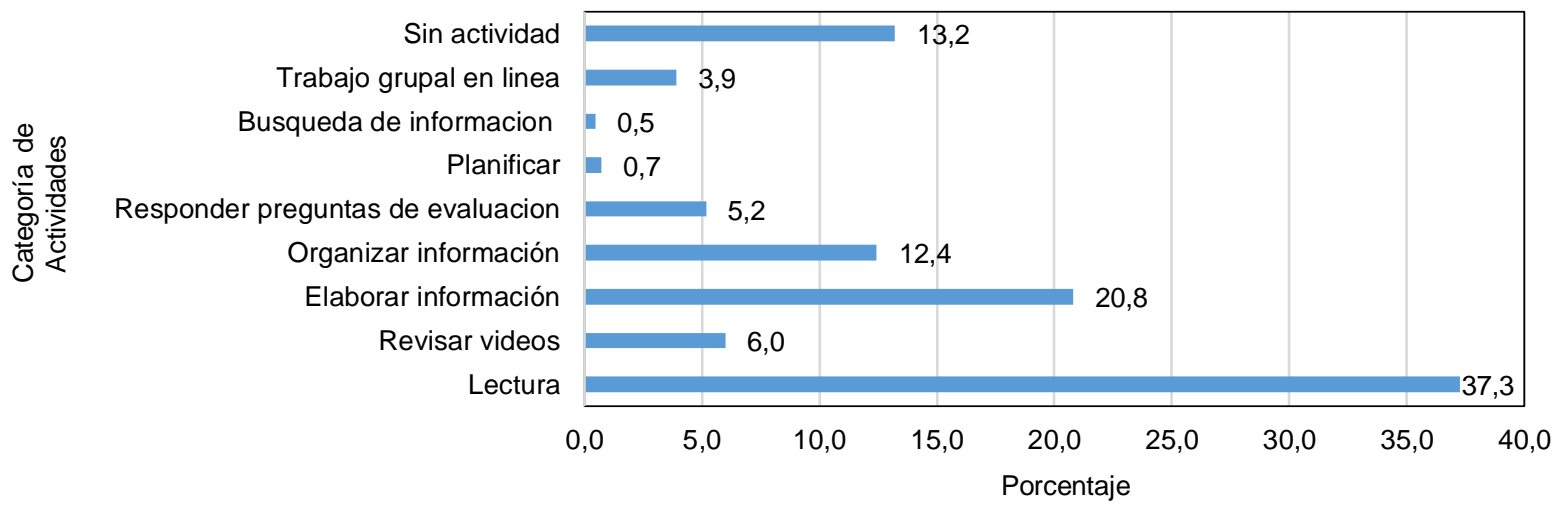

Fig. 8: Porcentajes promedio por categoría de actividad de estudio a la semana 
En la figura 8 se observa, por un lado, que las actividades que predominan son: lectura, elaborar información y organizar información, actividades de estudio tradicionalmente desarrolladas para adquirir los conocimientos. Por otro lado, las actividades: revisar videos, responder preguntas de evaluación, trabajo grupal en línea, planificar y búsqueda de información no superan el $6 \%$ del porcentaje de tiempo promedio de estudio a la semana.

\section{Cantidad de asignaturas estudiadas}

La última variable analizada es la cantidad de asignaturas estudiadas por día. Se observa en la Figura 9 que los estudiantes en promedio indican estudiar como máximo dos asignaturas al día los lunes y martes. Así en el gráfico se puede observar la tendencia de asignaturas estudiadas durante cada día de una semana, que en promedio se dirige a la baja durante el fin de semana. Por lo anterior, se podría sugerir que los estudiantes mantienen un orden en una agenda semanal privilegiando otras actividades durante el fin de semana.

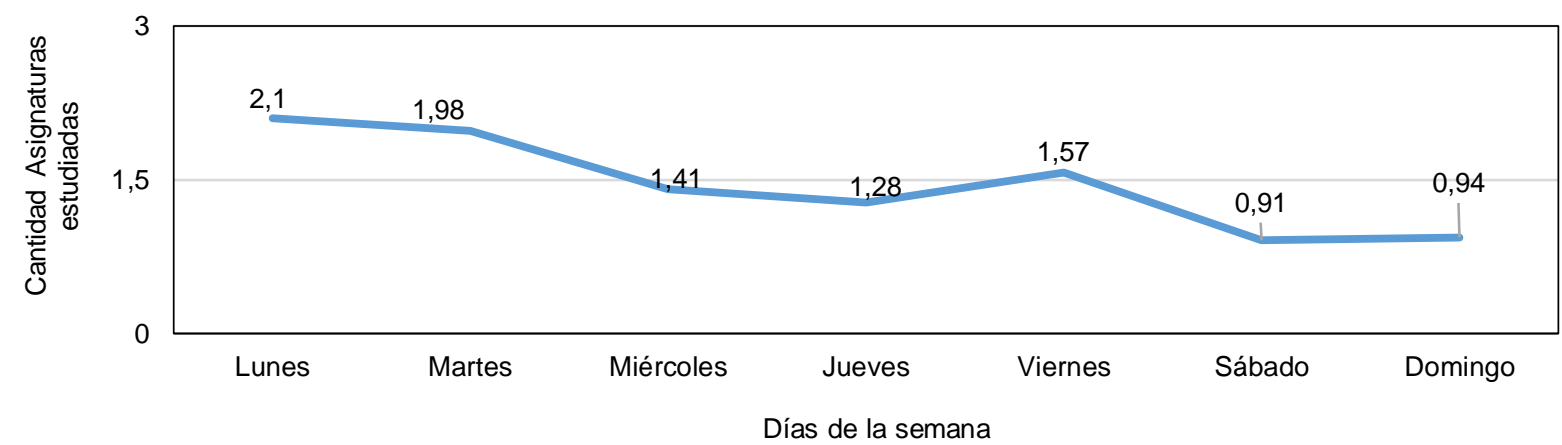

Fig. 9: Cantidad promedio de asignaturas a las que los estudiantes dedican tiempo por día

\section{DISCUSIÓN}

Se han presentado los resultados de una investigación preliminar que tuvo como objetivo estudiar la planificación del tiempo académico asincrónico de una muestra de estudiantes universitarios que pertenecen al grupo mejor evaluado. En este contexto, la importancia de la gestión del tiempo se centra en que, si un estudiante logra planificar y organizar bien su tiempo de estudio, es probable que cumpla con las tareas académicas (Rowe y Rafferty, 2013; Van Laer y Elen, 2017). En caso contrario, la posibilidad de que deje para último momento las tareas y que se desmotive lo podrían llevar a bajos desempeños académicos.

En relación con la primera pregunta de investigación que señala ¿cómo planifican su tiempo académico asincrónico una muestra de estudiantes universitarios que pertenecen al grupo mejor evaluado cuando se presentan condiciones de educación a distancia? Los resultados sugieren una tendencia de habito de estudio favorable que mostró que realizan actividades de estudio de forma permanente durante todos los días de la semana, estudiando alrededor de tres horas en promedio de lunes a viernes de forma autónoma e independiente a actividades que podrían ser obligatorias en las plataformas online. Esta situación se asocia a que posiblemente el perfil del estudiante que participó en el estudio podría tener una mejor autoorganización de su actividad académica quizás por un ambiente favorable al estudio, lo que coincide con otros estudios (Rowe y Rafferty, 2013; Van Laer y Elen, 2017). No obstante, la planificación como actividad declarada tiene una frecuencia muy baja de $0,7 \%$ del tiempo que dedican a la semana lo que coincide con otros estudios tales como (Zambrano et al, 2018) en que se reporta poca planificación del estudio.

También en relación con el tiempo de estudio a la semana, se mostró que los estudiantes de primer año son los que más tiempo señalaron dedicar al estudio asincrónico con $M=21,8$ horas a la semana versus estudiantes de último año en que disminuye a la mitad el tiempo de estudio asincrónico con $\mathrm{M}=10,2$ horas a la semana. Este resultado coincide con (Vosniadou, 2020; entre otros) quién destaca el rol de la autorregulación del aprendizaje como un puente entre la enseñanza secundaria y la educación superior como una opción para la optimización y gestión del tiempo académico. No obstante, es importante pensar y revisar si la sola gestión del tiempo puede ayudar a construir las habilidades de aprendizaje que se requieren hoy en día producto de la aceleración en la digitalización de la educación producto de la COVID-19 (Cabero, 2020).

Respecto a la segunda pregunta de investigación ¿qué actividades de estudio realizan?, se evidencio que las actividades de estudio que predominan son la lectura, seguida por la elaboración de información y posteriormente la organización de información. Al contrario, las actividades que mostraron una menor frecuencia de aparición son el trabajo grupal en línea y la actividad de planificar. La baja frecuencia de la actividad de planificación puede deberse a que no es necesario planificar ya que las actividades de estudio 
que ejecutan son actividades de estudio tradicionales y que no requieren de un mayor proceso de planificación. La situación anterior coincide con la baja frecuencia de actividades de búsqueda de información adicional al material o contenidos entregados por el profesor.

En este contexto, se advierten procesos reproductivos del conocimiento y centrados en la enseñanza, temas ampliamente estudiados por otras investigaciones (Löffler et al, 2011; entre otros). Debido a que se advierten procesos reproductivos del conocimiento y centrados en la enseñanza, se sugiere la inclusión en las clases online de estrategias de evaluación formativa tales como la aplicación de rubricas globales y/o analíticas (Manzanares y Arreba, 2014; Panadero y Jonsson, 2013), procedimientos que utilicen bitácoras y/o guías de aprendizaje (Zambrano, 2016) y retroalimentación que orientan la actividad de estudio de los estudiantes hacia el aprendizaje. No obstante, las estrategias anteriores requieren de un mayor tiempo por parte de los profesores online para realizar procesos de evaluación para el aprendizaje. Por ello, se requiere también de nuevos cálculos del tiempo del profesor online bajo condiciones de pandemia y educación digital. Así, el trabajo conjunto hacia el aprendizaje podría influir en la orientación hacia metas de aprendizaje (CovarrubiasApablaza et al, 2019).

Para la tercera pregunta de investigación ¿qué propósitos de estudio tienen? se constató poco tiempo para la preparación del estudio lo que sugiere que en la etapa de disposición al estudio no aplican o aplican muy poco el proceso de análisis de tareas (Zimmerman y Labuhn, 2012), dado que desarrollan directamente la actividad de estudio asincrónica. La preparación al estudio se refiere al momento en que el estudiante se activa para estudiar, analiza lo que tiene que hacer y prepara su ambiente para estudiar. El bajo porcentaje de tiempo destinado a la preparación del estudio se podría asociar con el tipo de actividad que declaran realizar que son procesos reproductivos del conocimiento que no requieren de mayor tiempo de preparación. En este contexto, se advierte la necesidad de una transformación de la educación que se acelera por la pandemia de Covid-19 (Cabero, 2020).

Finalmente, se menciona que el instrumento aplicado en el estudio es de utilidad para ser usado por los docentes en el aula virtual y también para los estudiantes para su propio autocontrol. Por ello, se aporta información valiosa para la planificación, implementación y evaluación de las actividades educativas a distancia. Respecto a las limitaciones del estudio estas son: el tamaño de la muestra que consideró a 73 sujetos que cumplían con el criterio de pertenecer al 15\% mejor evaluado durante el primer semestre del año 2020. No obstante, esta limitación puede ser remediada en futuras investigaciones dado el carácter exploratorio de este estudio.

\section{CONCLUSIONES}

Las conclusiones de este estudio son:

1. En relación con la planificación del tiempo académico de estudio asincrónico se advierte poco tiempo para la preparación del estudio y poca autorregulación en la etapa de disposición al estudio, dado que van directo a la realización de la actividad asincrónica.

2. Respecto a las actividades de estudio que predominan son: lectura, elaboración de información y organización de información. La actividad de reflexión respecto a lo que se estudia y los resultados no se observó en los registros de los estudiantes. Actividades como el trabajo grupal en línea y la planificación muestran una frecuencia muy menor de aparición. Asimismo, el registro de planificación utilizado como instrumento de medida también puede ser usado por los estudiantes como un proceso de autogestión y para su propia metacognición.

3. En síntesis, se sugiere orientar el trabajo de docencia online hacía la inclusión de estrategias de evaluación formativa, la planificación y gestión del tiempo de los estudiantes con conciencia propia de ellos y del impacto que puede tener comprender lo que es aprender.

\section{AGRADECIMIENTOS}

Proyecto ANID-COVID 1012 "Desarrollo e implementación de procedimientos docentes para facilitar la disposición al aprendizaje en condiciones de distanciamiento físico por pandemia de Covid-19, en asignaturas de primer año universitario con mediano o alto riesgo de fracaso".

\section{REFERENCIAS}

Andréu, J., Las Técnicas de Análisis de Contenido: Una Revisión Actualizada. Documento de Trabajo, S2001/03, Centro de Estudios Andaluces, (2001)

Baars, M. A., Nije Bijvank, M., Tonnaer, G. H. y Jolles, J. Self-report Measures of Executive Functioning are a Determinant of Academic Performance in First-year Students at a University of Applied Sciences. https://doi.org/ 10.3389/fpsyg.2015.01131, Frontiers in Psychology, 6, 1131 (2015) 
Bellei, C., Orellana, V. y Canales, M., Elección de Escuela en la Clase Alta Chilena. Comunidad, Identidad y Cierre Social. https://doi.org/10.14507/epaa.28.3884, Archivos Analíticos de Políticas Educativas, 28(5), 1-22 (2020)

Bellei, C., Canales, M., Orellana, V. y Contreras, M., Elección de Escuela en Sectores Populares: Estado, Mercado e Integración Social, https://doi.org/10.4206/rev.austral.cienc.soc.2016.n31-06, Revista Austral de Ciencias Sociales, 31, 91-110 (2016)

Boekaerts, M. y Corno, L. Self-regulation in the Classroom: A Perspective on Assessment and Intervention. https://doi.org/10.1111/j.1464-0597.2005.00205.x, Applied Psychology, 54(2), 199-231 (2005)

Cabero, J. Aprendiendo del Tiempo de la COVID-19, https://doi.org/10.15359/ree.24-s.2, Revista Electrónica Educare, 24, 9 (2020)

Canales, M., Bellei, C. y Orellana, V., ¿Por Qué Elegir una Escuela Privada Subvencionada? Sectores Medios Emergentes y Elección de Escuela en un Sistema de Mercado. https://doi.org/10.4067/S0718-07052016000400005, Estudios pedagógicos, 42(3), 89- 109 (2016)

Covarrubias-Apablaza, C. G., Acosta-Antognoni, H., \& Mendoza-Lira, M. Relación de autorregulación del aprendizaje y autoeficacia general con las metas académicas de estudiantes universitarios. Form. Univ., 12(6), 103-114 (2019)

Delen, E., Liew, J. y Willson, V. Effects of Interactivity and Instructional Scaffolding on Learning: Self-regulation in Online Video-based Environments, https://doi.org/10.1016/j.compedu.2014.06.018, Computers \& Education, 78, 312-320 (2014)

Fokkens-Bruinsma, M., Vermue, C., Deinum, J. F y Van Rooij, E. First-year Academic Achievement: The Role of Academic Self-efficacy, Self-regulated Learning and Beyond Classroom Engagement, https://doi.org/10.1080/02602938.2020.1845606, Assessment \& Evaluation in Higher Education, 1-12 (2020)

García-Marcos, C. J., López-Vargas, O. y Cabero-Almenara, J. Autorregulación del Aprendizaje en la Formación Profesional a Distancia: Efectos de la Gestión del Tiempo, https://doi.org/10.6018/red.400071, Revista de Educación a Distancia, 20(62) (2020)

Liaw, S. y Huang, H. Perceived Satisfaction, Perceived Usefulness and Interactive Learning Environments as Predictors to Self-regulation in E-learning Environments. https://doi.org/10.1016/j.compedu.2012.07.015, Computers \& Education, 60(1), 14-24 (2013)

Löffler, S., Feja, C., y otros cuatro autores, K. Interactive versus Reproductive Learning, a Comparison of Medical School Graduates with Participants of a Postgraduate CPD Session, https://doi.org/10.3205/zma000769GMS, Zeitschrift für Medizinische Ausbildung, 28(4) (2011)

Manzanares, M. y Arreba, A. Aprendizaje Basado en la Evaluación mediante Rúbricas en Educación Superior. https://doi.org/10.1016/S0121-4381(14)70004-9, Suma Psicológica, 21(1), 28-35 (2014)

Hadwin, A. y Oshige, M. Socially Shared Regulation: Exploring Perspectives of Social in Self-Regulated Learning Theory. https://doi.org/10.4324/9780203839010, Teachers College Record, 113(2), 240-264 (2011)

Panadero, E. A Review of Self-regulated Learning: Six Models and Four Directions for Research. https://doi.org/10.3389/fpsyg.2017.00422, Frontiers in Psychology, 8, 422 (2017)

Panadero, E., y Jonsson, A. The Use of Scoring Rubrics for Formative Assessment Purposes Revisited: A Review. https://doi.org/10.1016/j.edurev.2013.01.002, Educational Research Review, 9, 129-144 (2013)

Pérez-Villalobos, M. V., Cobo-Rendón, R. C., Sáez, F. M. y Díaz-Mujica, A. E. Revisión Sistemática de la Habilidad de Autocontrol del Estudiante y su Rendimiento Académico en la Vida Universitaria, https://doi.org/10.4067/S071850062018000300049, Formación Universitaria, 11(3), 49-62 (2018)

Pintrich, P. The Role of Goal Orientation in Self-regulated Learning. In Handbook of Self-Regulation, 1era edición, 452502, Academic Press, San Diego, EE. UU (2000)

Puustinen, M. y Pulkkinen, L. Models of Self-regulated Learning: A Review. https://doi.org/10.1080/00313830120074206, Scandinavian Journal of Educational Research, 45(3), 269-286 (2001)

Rowe, F. y Rafferty, J. Instructional Design Interventions for Supporting Self-regulated Learning: Enhancing Academic Outcomes in Postsecondary E-learning Environments. Journal of Online Learning and Teaching, 9(4), 590-601 (2013)

Van Laer, S y Elen, J. In Search of Attributes that Support Self-regulation in Blended Learning Environments. https://doi.org/10.1007/s10639-016-9505-x, Education and Information Technologies, 22(4), 1395-1454 (2017)

Vosniadou, S. Bridging Secondary and Higher Education. The Importance of Self-regulated Learning. https://doi.org/10.1017/S1062798720000939, European Review, 28(S1), S94-S103 (2020)

Zambrano, C. Autoeficacia, Prácticas de Aprendizaje Autorregulado y Docencia para Fomentar el Aprendizaje Autorregulado en un Curso de Ingeniería de Software. https://doi.org/10.4067/S0718-50062016000300007, Formación Universitaria, 9(3), 51-60 (2016)

Zambrano, C., Albarran, F., \& Salcedo, P. A. Percepción de Estudiantes de Pedagogía respecto de la Autorregulación del Aprendizaje. https://dx.doi.org/10.4067/S0718-50062018000300073, Formación Universitaria, 11(3), 73-86 (2018)

Zimmerman, B. J. y Labuhn, A. S. Self-regulation of Learning: Process Approaches to Personal Development. In APA Educational Psychology Handbook: Theories, Constructs, and Critical Issues, 1era edición, 399-425. American Psychological Association, Washington, EE. UU (2012) 